\title{
Hyperuricemia in psoriatic arthritis: prevalence and associated factors
}

\author{
Tania Gudu ${ }^{1,2}$, Alexandra Peltea ${ }^{1,2}$, Andra Balanescu ${ }^{1,2}$, Violeta Bojinca ${ }^{1,2}$, \\ Daniela Opris-Belinski ${ }^{1,2}$, Denisa Predeteanu ${ }^{1,2}$, Ruxandra Ionescu ${ }^{1,2}$ \\ ${ }^{1}$ Department of Rheumatology and Internal Medicine, "Sf. Maria" Hospital, Bucharest, Romania \\ 2"Carol Davila" University of Medicine and Pharmacy, Bucharest, Romania
}

\begin{abstract}
Hyperuricemia is frequent in psoriatic arthritis (PsA) and it seems to be related to metabolic syndrome rather than extensive psoriatic skin disease. The objectives of this study were to evaluate the prevalence of hyperuricemia in PsA patients and to identify the associ-ated factors.

Design: cross-sectional study, including consecutive, unselected, adult PsA patients. Data collection: demographic variables (age, gender, disease duration), clinical variables (affected joints, current moderate/severe psoriasis, nail disease, axial involvement, enthesi-tis, dactylitis), laboratory variables (acute phase reactants), treatment-related variables (non-steroidal anti-inflammatory drugs, corticosteroids, synthetic and biologic disease modifying drugs) and comorbidities. Hyperuricemia was defined as uric acid level above $6.8 \mathrm{mg} / \mathrm{dl}$. Statistical analysis: the factors that were potentially associated with hyperuricemia were assessed by uni- and multivariate logistic regression.

In all, 120 PsA patients were included in the study: 69 (57.5\%) women, mean age \pm standard deviation $54 \pm 11.8$ years, mean disease duration $7 \pm 7.4$ years; $24(20 \%)$ had moderate/severe psoriasis and $30(25 \%)$ were taking a biologic. Around a quarter of patients had hyperuricemia (33; $27.5 \%)$. Hyperuricemia was significantly associated with obesity, diabetes, ischemic heart disease and hypertension, but there was no correlation with current skin psoriasis. In the multivariate analysis, it was best explained by diabetes (odds ratio: 4.95, [95\% confidence intervals: $1.47 ; 16.67])$, ischemic heart disease $(3.61[1.00 ; 12.98])$ and obesity $(1.86[1.04 ; 3.32])$.

Hyperuricemia in PsA is associated with metabolic syndrome rather than skin dis-ease, but further longitudinal studies are needed to identify causal relationships.
\end{abstract}

Keywords: psoriatic arthritis, hyperuricemia, moderate/severe psoriasis, metabolic syndrome

\section{INTRODUCTION}

Psoriatic arthritis (PsA) is a chronic inflammatory disease which is associated with skin psoriasis. Studies have demonstrated associations between PsA and multiple comorbidities, in particular cardiovascular risk factors and diseases (1-5). Although little studied, the relationship between PsA and hyperuricemia is well-known for a long time (6). The prevalence of hyperuricemia in PsA is reported to be around $20 \%$ (7) and the main proposed cause was the extension of psoriasis lesions, through an increased cellular turn-over (8). However, more recent studies have suggested that hyperuricemia in PsA and in psoriasis also is due to the association with the metabolic syndrome and cardiovascular comor- bidities, in particular obesity and hyperlipidaemia (7, 9-11).

The objective of this study was to evaluate the prevalence of hyperuricemia in PsA patients and to identify the associated factors.

\section{METHODS}

\section{Study design and patients}

The study had a cross-sectional design and included unselected, consecutive adult patients with definite PsA according to the physician, with a range of disease manifestations and treatments. Most of them fulfilled the CASPAR classification criteria for PsA [12]. All patients gave a written informed con- 
sent and the protocol of the study was approved by the local ethics committee.

\section{Data collection}

Several variables, i.e., demographic, clinical, laboratory variables and data on comorbidities and treatment were collected. Demographic features included age, gender and disease duration. Diseaserelated characteristics included swollen joint count, SJC (0-66), tender joint count, TJC (0-68), current moderate or severe psoriasis, i.e., more than $5 \%$ body surface, nail disease, other activity such as dactylitis, enthesitis and axial involvement, and acute phase reactants, e.g., C-reactive protein (CRP) and erythrocyte sedimentation rate (ESR). Data regarding treatment included current and past non-steroidal anti-inflammatory drugs (NSAIDs), corticosteroids, synthetic and biologic disease modifying drugs (sDMARDs, b DMARDs, respectively). Data on comorbidities were also collected, according to the recent European League Against Rheumatism (EULAR) recommendations on reporting, screening and preventing comorbidities in chronic inflammatory rheumatic diseases (13). This included cardiovascular diseases (i.e., history of myocardial infarction, pectoris angina, stent, stroke, transient ischemic attack, heart failure and lower limb peripheral arterial disease), malignancies (i.e., any history of malignancy), infections (i.e., history of tuberculosis, of serious infections, opportunistic infections and chronic viral infections), gastro-intestinal diseases (i.e., history of peptic ulcer), osteoporosis and depression. Risk fac- tors and current treatment for comorbid conditions were also collected.

\section{Statistical analysis}

Hyperuricemia was defined as serum uric acid level above $6.8 \mathrm{mg} / \mathrm{dl}$ (14) since it was shown that this level defines the saturation point of the sodium urate salt in biologic fluids at physiologic $\mathrm{pH}$ and temperature (15).

Associations between hyperuricemia and other variables were tested by Spearman's correlation (statistical significance: $\mathrm{p}<0.05$ ). Factors associated to hyperuricemia were identified by performing univariate and multivariate logistic regressions. The variables tested were: age, gender, disease duration, SJC, TJC, CRP, ESR, current moderate/sever psoriasis, nail disease, other activity (dactylitis, enthesitis, axial involvement), current NSAIDs, corticosteroids, sDMARDs and bDMARDs and comorbidities and risk factors, as described above. Only variables that were statistically significant associated to hyperuricemia in the univariate logistic regression $(p<0.10)$ were included in the multivariate analysis.

There was no imputation of missing data. Statistics were performed using SPPS for IBM, version 20.0 .

\section{RESULTS}

\section{Patient characteristics}

A total of 120 PsA patients were analysed (Table 1). Mean \pm standard deviation age was $54 \pm 11.8$ years,

TABLE 1. Characteristics of 120 patients with psoriatic arthritis, according to the presence of hyperuricemia

\begin{tabular}{|c|c|c|c|}
\hline Patient characteristics & $\begin{array}{l}\text { All patients } \\
(\mathrm{N}=120)\end{array}$ & $\begin{array}{c}\text { Patients with } \\
\text { hyperuricemia }(\mathrm{N}=33)\end{array}$ & \begin{tabular}{|c|} 
Patients with \\
normouricemia $(\mathrm{N}=87)$
\end{tabular} \\
\hline Females, N (\%) & $69(57.5)$ & $15(45.5)$ & $54(62)$ \\
\hline Age, years, mean (SD) & $54(11.8)$ & 56.5 (10.9) & $53.1(12.1)$ \\
\hline PsA disease dura on, years, mean (SD) & $7.2(7.5)$ & $5.5(5.7)$ & $7.8(8.1)$ \\
\hline Swollen joint count (0-66), median (range) & $0(0,9)$ & $0(0,4)$ & $0(0,9)$ \\
\hline Tender joint count (0-68), median (range) & $2(0,22)$ & $2(0,14)$ & $2(0,22)$ \\
\hline $\mathrm{CRP}, \mathrm{mg} / \mathrm{L}$, median (range) & $7(0,121)$ & $6.5(0,65)$ & $7.4(0,21)$ \\
\hline Current moderate/ severe skin psoriasis, $\mathrm{N}(\%)$ & $24(20)$ & $7(29.2)$ & $17(70.8)$ \\
\hline Enthesi s, N (\%) & $18(15)$ & $7(38.9)$ & $11(61.1)$ \\
\hline Dactyli s, N (\%) & $31(25.8)$ & $8(25.8)$ & $23(74.2)$ \\
\hline Obesity, N (\%) & $41(34.2)$ & $18(54.5)$ & $23(26.4)$ \\
\hline Hypertension, $\mathrm{N}(\%)$ & $62(51.7)$ & $22(66.7)$ & $40(46.5)$ \\
\hline Diabetes, N (\%) & $18(15)$ & $11(33.3)$ & $7(8.0)$ \\
\hline Ischemic heart disease, $\mathrm{N}(\%)$ & $15(12.5)$ & $8(4.22)$ & $7(8.0)$ \\
\hline Biologic drug, N (\%) & $30(25)$ & $8(26.7)$ & $22(73.3)$ \\
\hline
\end{tabular}

SD: standard deviation; PsA: psoriatic arthritis; CRP: C-reactive protein 
mean disease duration was $7.2 \pm 7.5$ years and the majority were females (57.5\%). Twenty four patients (20\%) presented current moderate/severe skin psoriasis. Most of them (78.3\%) were treated with a synthetic DMARD and about a quarter $(25 \%)$ with a biologic drug. Many patients had associated comorbidities, the cardiovascular diseases and risk factors being the most prevalent: dyslipidaemia $80 \%$, hypertension $51.7 \%$, obesity $34.2 \%$, and cardiovascular events $34.2 \%$.

\section{Hyperuricemia and associated factors}

Around a third of the patients (27.5\%) had hyperuricemia. There were no differences in PsA patients with a high level of serum uric acid compared to the ones with normal levels with respect to demographic and clinical variables (Table 1). However, patients with hyperuricemia had a higher prevalence of cardiovascular comorbidities, namely obesity, hypertension, ischemic heart disease and diabetes (Table 1).

Hyperuricemia correlated only with cardiovascular comorbidities, including obesity $(\mathrm{r}=0.296$, $\mathrm{p}=0.001)$, diabetes $(\mathrm{r}=0.221, \mathrm{p}=0.015)$, ischemic heart disease $(\mathrm{r}=0.219, \mathrm{p}=0.016)$ and hypertension $(\mathrm{r}=0.181, \mathrm{p}=0.049)$, but not with moderate/severe skin psoriasis $(\mathrm{r}=0.016, \mathrm{p}=0.862)$. The variables that were statistically significant associated to hyperuricemia in the univariate logistic regression and then included in the multivariate analysis were: obesity, hypertension, chronic kidney disease, diabetes and ischemic heart disease (Table 2). In the multivariate analysis, hyperuricemia was best explained by diabetes (odds ratio: 4.95, [95\% confidence intervals: $1.47 ; 16.67])$, ischemic heart disease (3.61 [1.00; 12.98]) and obesity (1.86 [1.04; 3.32]) (Table 2).

TABLE 2. Factors associated with hyperuricemia in psoriatic arthritis: univariate and multivariate logistic regressions

\begin{tabular}{|l|c|c|}
\hline Variable & $\begin{array}{c}\text { Univariate } \\
\text { analysis p value }\end{array}$ & $\begin{array}{c}\text { Multivariate analysis } \\
\text { OR [95\% confidence } \\
\text { interval] (p value) }\end{array}$ \\
\hline Obesity & 0.001 & $\begin{array}{c}1.86[1.04 ; 3.32] \\
(0.03)\end{array}$ \\
\hline Hypertension & 0.049 & $\begin{array}{c}1.17[0.42 ; 3.30] \\
(0.76)\end{array}$ \\
\hline $\begin{array}{l}\text { Chronic kidney } \\
\text { disease }\end{array}$ & 0.098 & $\begin{array}{c}0.28[0.03 ; 1.48] \\
(0.12)\end{array}$ \\
\hline $\begin{array}{l}\text { Diabetes mellitus } \\
\text { Ischemic heart } \\
\text { disease }\end{array}$ & 0.001 & $\begin{array}{c}4.95[1.47 ; 16.67] \\
(0.01)\end{array}$ \\
\hline
\end{tabular}

For the multivariate analysis, significant results are in bold type

\section{DISCUSSION}

The study confirms that hyperuricemia is frequent in PsA, since almost a third of the patients had high levels of serum uric acid. Moreover, it was best explained by some of the cardiovascular comorbidities and not by the extent of the skin lesions.

Hyperuricemia occurred in $27.5 \%$ of the PsA patients, similar to previous studies (7). The prevalence is higher than in the general population where hyperuricemia is reported to occur in 5 to $13 \%$ (16). This is probably due to the association with the metabolic syndrome, which was demonstrated to be higher in PsA compared to the general population $(1,5)$.

The factors that were associated with hyperuricemia in the current studies were mainly related to cardiovascular comorbidities; there was no relationship with the extent of the skin disease. These results are in concordance with previous studies. In psoriasis alone, hyperuricemia was also shown to have a higher prevalence than in the general population and to be associated with obesity and hypercholesterolemia rather than skin involvement $(10,17)$. In psoriatic arthritis there was also no correlation between serum uric acid and psoriasis extent. In a Canadian cohort of PsA patients, hyperuricemia was associated with age, hypertension, ischemic heart disease, vasodilators, elevated serum creatinine and elevated serum total cholesterol (7); however body mass index/ obesity was not included. The best predictive model that explained hyperuricemia in PsA in this study included renal impairment, namely high levels of serum creatinine (odds ratio, OR $30.8, \mathrm{p}<0.001$ ) and hypercholesterolemia (OR 3.7, p<0.001) (7).

The current study has several limitations. Firstly, it included PsA patients from a tertiary centre which may lead to a selection of more severe cases. However, the patient and disease characteristics were as expected. Secondly, the cross-sectional design of the study does not allow identifying causal relationships, only associations. Lastly, is very difficult to have a standardized screening for comorbidities and risk factors in clinical practice. However, in the current study all PsA patients were screened for cardiovascular diseases.

In clinical practice is important to determine the level of serum uric acid in PsA patients, since high values might suggest a possible association with the metabolic syndrome, and therefore a screening for cardiovascular comorbidities should be done. In PsA there is a high prevalence of cardiovascular comor- 
bidities, more than in the general population and psoriasis and similar to diabetes or rheumatoid arthritis $(1,2,5,18)$. This is due to both traditional and non-traditional risk factors $(1,2)$. The importance of cardiovascular screening in PsA is also supported by the EULAR recommendations for the management of cardiovascular in inflammatory arthritis (19). Furthermore, the evaluation of comorbidities including cardiovascular diseases was stated as an overarching principle in both EULAR and Group for Research and Assessment of Psoriasis and Psoriatic Arthritis
(GRAPPA) recommendations for management of PsA $(20,21)$. Even in the absence of manifest cardiovascular diseases or risk factors, a special attention to these comorbidities is still needed, since hyperuricemia was shown to be correlated to subclinical atherosclerosis in PsA (2).

In conclusion, hyperuricemia in PsA is associated with metabolic syndrome rather than skin disease, but further longitudinal studies are needed to identify causal relationships.

Conflict of interest: none declared Financial support: none declared

\section{REFERENCES}

1. Jamnitski A., Symmons D., Peters M.J., Sattar N., McInnes I., Nurmohamed M.T. Cardiovascular comorbidities in patients with psoriatic arthritis: a systematic review. Ann Rheum Dis 2013; 72(2):211-6

2. Husni M.E., Mease P.J. Managing comorbid disease in patients with psoriatic arthritis. Curr Rheumatol Rep 2010;12(4):281-7

3. Ogdie A., Yu Y., Haynes K., Love T.J., Maliha S., Jiang Y., Troxel A.B., Hennessy S., Kimmel S.E., Margolis D.J., Choi H., Mehta N.N., Gelfand J.M. Risk of major cardiovascular events in patients with psoriatic arthritis, psoriasis and rheumatoid arthritis: a population-based cohort study. Ann Rheum Dis 2015; 74(2):326-32.

4. Husted J.A., Thavaneswaran A., Chandran V., Eder L., Rosen C.F., Cook R.J., Gladman D.D. Cardiovascular and other comorbidities in patients with psoriatic arthritis: a comparison with patients with psoriasis. Arthritis Care Res (Hoboken) 2011; 63(12):1729-35

5. Gladman D.D., Ang M., Su L., Tom B.D., Schentag C.T., Farewell V.T. Cardiovas-cular morbidity in psoriatic arthritis (PsA). Ann Rheum Dis 2009; 68:1131-5

6. Eisen A.Z., Seegmiller J.E. Uric acid metabolism in psoriasis. J Clin Invest 1961;40:1486-94

7. Bruce I.N., Schentag C.T., Gladman D.D. Hyperuricemia in psoriatic arthritis: prevalence and associated features. J Clin Rheumatol 2000; 6(1):6-9.

8. Kizaki H., Matsuo I., Sakurada T. Xanthine oxidase and guanase activities in normal and psoriatic epidermis. Clin Chim Acta 1977; 75(1):1-4.

9. Scott J.T., Stodell M.A. Serum uric acid levels in psoriasis. Adv Exp Med Biol 1984; 165 Pt A:283-5.

10. Peserico A., Zanetti G., Padovan S., Bertoli P., Fornasa C.V., Cipriani R., Ambrosio G.B., Zamboni S., Pagnan A. Relationship between body weight and blood pressure and some metabolic parameters in psoriatic patients. Br J Dermatol 1988; 118(2):191-4

11. Gisondi P. Hyperuricemia in patients with chronic plaque psoriasis. Drug Dev Res 2014;75 Suppl 1:S70-2

12. Chandran V., Schentag C.T., Gladman D.D. Sensitivity of the classification of psoriatic arthritis criteria in early psoriatic arthritis. Arthritis Rheum 2007; 57:1560-3
13. Baillet A., Gossec L., Carmona L., Wit M.D., van Eijk-Hustings Y., Bertheussen H. et al. Points to consider for reporting, screening for and preventing selected comorbidities in chronic inflammatory rheumatic diseases in daily practice: a EULAR initiative. Ann Rheum Dis 2016; 75(6):965-73

14. Campion E.W., Glynn R.J., DeLabry L.O. Asymptomatic hyperuricemia. Risks and consequences in the Normative Aging Study. Am J Med 1987; 82(3):421-6

15. Loeb J. The influence of temperature on the solubility of monosodium urate. Arthritis Rheum 1972;15:189-92

16. Klein R., Klein B.E., Cornoni J.C., Maready J., Cassel J.C., Tyroler H.A. Serum Uric Acidlts Relationship to Coronary Heart Disease Risk Factors and Cardiovascular Dis-ease, Evans County, Georgia. Arch Intern Med 1973;132(3):401-410

17. Brenner W., Gschnait F. Serum uric acid levels in untreated and PUVA-treated patients with psoriasis. Dermatologica 1978; 157(2):91-5

18. Han C., Robinson D.W. Jr, Hackett M.V. et al. Cardiovascular disease and risk factors in patients with rheumatoid arthritis, psoriatic arthritis, and ankylosing spondylitis. J Rheumatol 2006; 33:2167-2172.

19. Peters M.J., Symmons D.P., McCarey D., Dijkmans B.A., Nicola P., Kvien T.K., McInnes I.B., Haentzschel H., Gonzalez-Gay M.A., Provan S., Semb A., Sidiropoulos P., Kitas G., Smulders Y.M., Soubrier M., Szekanecz Z., Sattar N., Nurmohamed M.T. EULAR evidence-based recommendations for cardiovascular risk management in patients with rheumatoid arthritis and other forms of inflammatory arthritis. Ann Rheum Dis 2010; 69(2):325-31

20. Gossec L., Smolen J.S., Ramiro S., de Wit M., Cutolo M., Dougados M., Emery P. et al. European League Against Rheumatism (EULAR) recommendations for the management of psoriatic arthritis with pharmacological therapies: 2015 update. Ann Rheum Dis 2016; 75(3):499-510

21. Coates L.C., Kavanaugh A., Mease P.J., Soriano E.R., Laura Acosta-Felquer M., Armstrong A.W. et al. Group for Research and Assessment of Psoriasis and Psoriatic Arthritis 2015 Treatment Recommendations for Psoriatic Arthritis. Arthritis Rheumatol 2016; 68(5):1060-71 